\title{
NANOMEDICINE AND MAMMALIAN SPERM: LESSONS FROM THE PORCINE MODEL
}

Natalia Barkalina, MD, $\mathrm{MSc}^{1}$ (natalia.barkalina@obs-gyn.ox.ac.uk)

Celine Jones ${ }^{1}$ (celine.jones@obs-gyn.ox.ac.uk)

Kevin Coward, $\mathrm{PhD}^{1}$ (kevin.coward@obs-gyn.ox.ac.uk)

${ }^{1}$ Nuffield Department of Obstetrics and Gynaecology, University of Oxford, Level 3, Women's Centre, John Radcliffe Hospital, Headington, Oxford, OX3 9DU, UK;

Corresponding author (permanent address): Kevin Coward, $\mathrm{PhD}$, Nuffield Department of Obstetrics and Gynaecology, Level 3, Women's Centre, John Radcliffe Hospital, Headington, Oxford, OX3 9DU, United Kingdom (Telephone: +441865 782878; E-mail: kevin.coward@obsgyn.ox.ac.uk).

\section{ABSTRACT}

Biomedical nanotechnology allows us to engineer versatile nano-sized platforms that are comparable in size to biological molecules and intracellular organelles. These platforms can be loaded with large amounts of biological cargo, administered systemically and act at a distance, target specific cell populations, undergo intracellular internalisation via endogenous uptake mechanisms, and act as contrast agents or release cargo for therapeutic purposes. Over recent years, nanomaterials have been increasingly viewed upon as favourable candidates for intra-gamete delivery. In the case of sperm, nanomaterial-based approaches have been shown to particularly improve the efficacy of existing techniques such as sperm-mediated gene transfer, loading sperm with exogenous proteins, and tagging sperm for subsequent sex- or function-based sorting.

In this short review, we provide an outline of the current state of nanotechnology for biomedical applications in reproductive biology, and present highlights from a series of our studies evaluating the use of specialised silica nanoparticles in boar sperm as a potential delivery vehicle into mammalian gametes. This encouraging data, obtained from the porcine model in our 
laboratory, have formed the basis for the ethical approval of similar experiments in human sperm, thereby bringing us a step closer towards the potential use of this novel technology in the clinical environment.

Keywords: nanotechnology, nanomaterials, drug delivery, sperm, oocytes, embryos, assisted reproduction technology.

\section{BACKGROUND}

Reproductive science is an exciting and continuously developing field which aims to provide theoretical insight into the mechanisms of gametogenesis, fertilisation, early embryo development, pregnancy establishment, and to develop applied tools for the intentional manipulation of these processes to address fertility issues across different biological species. Assisted reproduction technology (ART), a collection of sophisticated laboratory techniques and approaches for the in vitro manipulation of gametes and embryos that were developed to enhance the chances of fertilisation and development to full-term, respectively, has become essential for the production of livestock [1-3], the preservation of animal species [4], and also for the treatment of human infertility $[5,6]$. In addition, reproductive science investigates an array of fundamental biological processes underlying reproduction, including cell division and fusion, genetic recombination, maintenance of pluripotency, and cell migration and differentiation, which are very similar to the events involved in tumour biogenesis and tissue regeneration. Therefore, research into reproductive biology has an inherent translational component, and permits advancement in our understanding of cell-based regenerative therapies for cardiovascular, renal, neurological and other diseases [7-9], and the fine mechanisms of cancer $[10,11]$. Over the last few decades, the field of reproductive science has expanded tremendously, now incorporating the principles of cell culture, micromanipulation, live cell microscopy, cryobiology, laser technology, proteomics, metabolomics, molecular genetics and biology. Such rapid development and the continuous introduction of novel techniques into this biologically and ethically sensitive field [12] requires thorough investigation of the potential risk/benefits ratio, including permanent effects upon offspring. Such investigation 
traditionally includes a fundamental step which evaluates short- and long-term safety and efficacy in a variety of both vertebrate and invertebrate animal models [13].

In this short review, we provide a brief outline of the current state of nanotechnology for biomedical applications in reproductive biology, and present highlights from a series of our studies evaluating the use of specialised silica nanoparticles in boar sperm as a potential delivery vehicle into mammalian gametes.

\section{NANOTECHNOLOGY AS A VERSATILE TOOL FOR TARGETED BIOLOGICAL DELIVERY AND ITS POTENTIAL USES FOR ART}

Nanotechnology is an innovative multifaceted discipline, combining the aspects of physics, chemistry, biology, mathematics, engineering and computer sciences, which designs and investigates the properties of small-scale (in general, 1-100nm) structures for the variety of industrial, consumer and scientific applications. In biomedicine, nanotechnology allows us to engineer versatile nano-sized platforms that are comparable in size to biological molecules and intracellular organelles. These platforms can be loaded with large amounts of biological cargo, administered systemically and act at a distance, target specific cell populations, undergo intracellular internalisation via endogenous uptake mechanisms, and act as contrast agents or release cargo for therapeutic purposes [14]. The ability to adjust the physical and surface chemical properties of nanomaterials, robustness, action at a distance, inherent 'affinity' towards malignant cells in whole-body models, along with the potential for additional active targeting, has resulted in the rapid expansion of nanomaterial-based technologies for drug delivery [15]. To date, nanomaterials have been demonstrated to facilitate selective delivery of a range of wellcharacterised and novel drugs into various target cells, including malignant lesions [16], immune cells [17], cells across the in vivo blood-tissue barriers, for example, the blood-brain barrier [18], and resistant bacterial cells $[19,20]$. Nanomaterials reduce the systemic toxicity of pharmacological agents, since they deliver cargo precisely into the cell population of interest, and allow the novel application of previously known, but rarely used, molecular compounds with unfavourable 
biodistribution, including small molecules [21], and agents for gene-based therapies such as DNA, mRNA, microRNA, small interfering RNA, and antisense oligonucleotides [22]. Nanomaterials have also been applied as contrast agents for the targeted early-stage detection of pathological lesions in whole-body models [23], and are capable of combining targeted detection and drug delivery capacity via the same platform, thus forming prototypes for highly adjustable personalised 'nanotheranostic' agents [24]. In future, these agents could be engineered to the needs of the individual patient, and could be administered systemically, target early stage pathological lesions and improve their visualisation during diagnostic imaging, and, at the same time, deliver therapeutic agents into affected cells under real-time control.

The revolutionary benefits of nanotechnology, especially from the perspective of improved intracellular delivery, have resulted in a growing number of studies applying the principles of nanomaterial-mediated delivery to help internalise molecular cargo into gametes [reviewed in 14, 25]. Indeed, studies of gamete structure and physiology across a variety of animal species can be compromised by the relatively poor physiological uptake capacity of mature sperm [26, 27] and oocytes [28] under in vitro conditions, which restricts the delivery of molecular probes into these cells unless aggressive membrane-permeating and fixation agents are applied [29-31]. Such techniques, interfering not only with the developmental capacity of gametes, but also with their viability, can severely restrict functional studies and downstream applications of treated gametes. Therefore, over recent years, nanomaterials have been increasingly viewed upon as favourable candidates for intra-gamete delivery, particularly in the case of sperm. Such approaches have improved the efficacy of existing techniques such as sperm-mediated gene transfer (SMGT) [3234], loading sperm with exogenous proteins while preserving their viability [35], and tagging sperm for subsequent sorting based upon sex chromosomes [36] or functional status [37]. Apart from the intracellular delivery angle, nanomaterials have also been used to tag preimplantation embryos [38], promote embryonic growth and development [39], and to assist in the bioimaging of sperm [40]. All such studies have been carried out in animal models, including the boar, bull, mouse and chicken. 


\section{LIVESTOCK SPERM AS A MODEL FOR NANOTECHNOLOGY STUDIES}

Interestingly, the majority of nanomaterial-mediated delivery studies in sperm have utilised cattle and pig sperm - a phenomenon with a rather simple explanation. As discussed previously, the use of assisted reproductive technology for breeding livestock is continuously expanding [1, 2, 4], and novel techniques are continuously being developed to improve efficacy. The increased demand for transgenic animals, both for livestock breeding and research purposes, and the inconsistent success rates of SMGT [41-44], which, otherwise, represents a straightforward and inexpensive alternative to micromanipulation techniques, has led to the clear need for increased sperm 'transfection' efficacy. Indeed some of the existing technologies are not readily applicable to livestock [45]. In order to address these issues, several independent research groups have attempted to use nanomaterials to load sperm with exogenous DNA [32-34]. For example, Kim et al. (2010) applied commercial magnetic iron nanoparticles and an external magnetic field ('magnitofection') to promote the uptake of $\mathrm{pCX}$-EGFP/Neo plasmid into boar sperm, and observed more than a 3-fold increase in the efficiency of this technique compared to simple co-incubation and sperm transfection with lipofectamine. In a further series of studies, Campos et al. (2011) showed that a commercial nanotransfectant and halloysite clay nanotubes were also effective in internalizing the pEGFP-N1 plasmid into bovine sperm.

Similarly, sex sorting of livestock sperm is an established methodology for animal breeding; however, the availability of photostable and more robust alternatives to conventional fluorochromes, which would be able to detect specific DNA sequences inside sperm, rather than quantify the total DNA amount inside these cells, could prove highly advantageous [46]. The most substantial amount of work in this direction has been associated with the development of specialised gold nanoparticles, with inherent strong optical properties, for sex sorting of bull sperm [36, 46-49]. In the most recent publication, Barchanski et al. (2015) applied polydisperse gold nanoparticles functionalized with locked nucleic acids (LNAs) and various cell-penetrating peptides as a prototype platform for the genetic labelling of bovine sperm, with encouraging outcomes. In 
another study, Odhiambo et al. (2013) used magnetic nanoparticles coated with anti-ubiquitin antibody, or lectin peanut agglutinin, for the 'nanopurification' of bull semen from defective and acrosome-damaged sperm, and demonstrated that insemination with nanopurified sperm increased conception rates.

Apart from addressing cutting-edge topics in ART for animal breeding, studies in animal models have, over the course of decades, allowed us to perform the essential screening of candidate technologies prior to their approval for pre-clinical testing in humans $[50,51]$. Although most fundamental studies of ART have traditionally utilised rodent models [52, 53], cattle and boar sperm have tended to be the model of choice for investigations involving nanomaterial-mediated delivery into human sperm. The choice of these particular models lies in a series of particularly beneficial characteristics. Firstly, sperm from these species are morphologically more similar to the paddle-shaped human sperm, as opposed to the hook-shaped sperm of rodents, and are more comparable in size. Given the versatile manner in which the physicochemical properties of nanomaterials can be adjusted, and the impact that such properties can exert upon binding and internalisation rates into target cells, the use of a model with closer structural resemblance is a very justified strategy. Secondly, livestock sperm for such experiments can be obtained via the acquisition of ejaculate, and therefore, are closer to human sperm used in the ART setting from the functionality and maturity perspective, in contrast to rodent sperm, which have to be retrieved surgically after the animal has been sacrificed. Thirdly, the acquisition of ejaculate can be carried out at designated livestock breeding facilities in parallel to their main routine activities, thus minimising the need for dedicated research-only husbandries. Finally, carrying out experiments in such models, in which the technologies being tested could prove advantageous per se, and likely to be highly sought after, is particularly beneficial from the research ethics perspective. 


\section{MESOPOROUS SILICA NANOPARTICLES AND BOAR SPERM: LESSONS LEARNT FROM IN VITRO STUDIES}

Since 2011, our group has been investigating the use of mesoporous silica nanoparticles (MSNPs) as a potential transfer vehicle into mammalian sperm and oocytes for ART. Over the course of our experiments, we have been viewing the technique of nanomaterial-mediated delivery as a potential cutting-edge addition to current assisted reproduction with numerous potential applications, spanning across the fields of fundamental reproductive biology, practical ART in veterinary science, and, perhaps, even to IVF in humans. This technology could have multiple uses for reproductive biology and medicine, including the improvement of SMGT efficiency, supplementation of sperm with specific molecules (deficiencies of which result in abnormal sperm function), fertilisation and early embryo development [54, 55], sperm sorting, delivery of 'nourishing' compounds into existing in vitro culture systems for oocytes and embryos, or tagging and tracking the fate of specific embryonic cell populations. All of these strategies could thus involve a simple-to-use chemical reagent which could be added to gametes and embryos in the IVF setting to exert desirable effects.

Our choice of MSNPs as a potential delivery tool into gametes was driven by the novelty and certain features of this nanomaterial, making at a promising candidate for reproductive applications, along with the limited existing data regarding its use in gametes in the ART environment [reviewed in 25]. MSNPs were introduced into biomedical applications in the early 2000s [56], and have been applied for targeted drug and gene delivery, bioimaging, and tissue engineering [57]. MSNPs originate from a special class of synthetically modified colloidal silica with highly regular mesopores, measuring from 2 to $50 \mathrm{~nm}$ in diameter [58]. Both particle size and pore diameter are adjustable via the modification of a surfactant-template base-catalysed sol-gel reaction, a traditional and universally preferred wet chemistry approach for MSNP synthesis, resulting in the production of particles with uniform sizes, which is essential for their controlled behaviour in biological systems [59-61]. At the same time, this inexpensive method of production 
can facilitate the in-house synthesis of MSNPs in various research laboratories, and thus help to promote their use. MSNPs are structurally robust, relatively inert chemically, and can carry larger amounts of payloads because of their porous nature, which markedly increases surface area [62]. MSNPs have low cytotoxicity, and a large body of evidence supports the safety and biocompatibility of this nanomaterial [63]. In addition, when we were designing our first experiments, mesoporous silica had already been consistently shown to exhibit strong delivery capacity, including the possibility to internalise nucleic into cells, acting as a non-viral gene delivery vector [64]. Collectively, all of these features contributed to our choice of MSNPs as a potential nanovector for reproductive biology: they appeared to have low cytotoxicity and did not promote the excessive formation of free radicals. Moreover, while representing an efficient delivery vehicle for various types of cargo, MSNPs could also be used as a stable cell tag.

The first stage of our experiments sought to evaluate the biocompatibility of MSNPs with gametes and provide the first insight into the possibility of applying this platform to these highly specialized cells [65]. In this set of experiments, we utilized boar sperm and mouse oocytes for the reasons extensively discussed in the previous section: these are not only considered as good models for human gametes during pre-clinical trials, but also represent important subjects of fundamental biology research themselves, in which the investigated technique could be used independently to improve the success rates of existing research methodologies. From the early stages, we specifically tried to gear the technique of MSNP-mediated delivery towards practical ART uses and view MSNPs as a customizable chemical reagent, which could be added to gametes under in vitro conditions to improve or modify their function. Therefore, we aimed to maintain our general research methodology, and experimental conditions, as similar as we could to those which would be used in a practical ART setting, particularly in terms of gamete handling procedures and exposure times. The first step of our investigation demonstrated that the simple in vitro exposure of boar sperm to MSNPs (Figure 1) with various surface chemistry in a $10-30 \mu \mathrm{g}$ per $10^{7}$ sperm ratio did not produce a negative effect upon total and progressive sperm motility, motion parameters, viability, 
acrosome morphology or DNA fragmentation index, as demonstrated by the results of computerassisted sperm analysis, viability staining, high-magnification phase-contrast microscopy and the sperm chromatin dispersion test, respectively. We also observed that during co-incubation, MSNPs could bind with various morphological regions of approximately one in five boar sperm (Figure 2). Stable binding rates were observed after just 2 hours of exposure, and were not affected by a subsequent increase of incubation time. MSNPs loaded with a nucleic acid or protein as prototypes for molecular cargo still bound to sperm, highlighting the possibility of simultaneous intracellular delivery. In an additional set of experiments, we demonstrated that MSNPs could also bind with the zona pellucida in mouse oocytes during in vitro exposure, although the spontaneous penetration of particles into oocytes was not evident. Nevertheless, the persistence of MSNPs in mouse oocytes for up to 24 hours after microinjection did not promote oocyte degradation (unpublished data), which, in our view, represented a very encouraging finding [25].

In our experiments, MSNPs showed binding with approximately $20 \%$ of sperm in a sample. However, interpretation of this finding in the context of existing literature was challenging, since the proportions of sperm associated with various nanoparticles following in vitro exposure has not been quantified before. Given the minimal effect of MSNP dose and exposure time upon binding rate, we hypothesised that binding could be restricted to a specific sperm population with certain functional parameters such as motility or viability. In an attempt to identify the potential effects of sperm functionality upon their 'affinity' to MSNPs, we applied a straightforward conventional discontinuous density gradient washing (DGW) technique to separate sperm into the 'better' and 'worse' quality sub-populations and assessed how differential sperm functionality in these two subpopulations might relate to their binding capacity to MSNPs [66]. As mentioned previously, the choice of DGW as a sperm selection method over other more sophisticated techniques, such as fluorescence-activated cell sorting (FACS), was driven primarily by our intention to maintain the practicality of MSNP-mediated technology for subsequent translation into a conventional ART setting. After subjecting MSNP-treated sperm to DGW, we were not able to detect significant 
differences between binding rate in the 'better' and 'worse' quality sperm sub-populations, which led to a hypothesis that MSNP binding with sperm occurs independently of the first-line parameters of sperm function, such as motility and viability, and could be related to the specific molecular structure or electrostatic charge of plasma membranes in certain sperm. Furthermore, we drew a conclusion that DGW, in its standard way, cannot be used to 'concentrate' MSNP-bound sperm and, therefore, alternative strategies are now required for the selection and enrichment of sperm populations with MSNP-bound cells for subsequent downstream applications.

In attempt to improve MSNP-sperm binding rates we also evaluated a universally deployed technique of nanoparticle targeting towards a specific cell population. For our experiments, we chose a specific cell-penetrating peptide, C105Y, previously characterised for its affinity towards mammalian sperm [27], as a promising targeting tool with receptor- and energy-independent internalisation into target cells [67]. These unique mechanisms of internalisation are highly advantageous for gametes, since they do require interaction with surface membrane receptors, many of which play key roles in gamete fusion and fertilisation. Inadvertent blockage of these receptors, could, therefore, have a negative effect upon fertilisation and thus restrict the potential application of nanoplatforms for reproductive biology. Targeting of MSNPs towards boar sperm with C105Y resulted in a significantly faster achievement of stable binding rates - after just 1 hour of incubation, approximately $20 \%$ to $30 \%$ of sperm showed signs of binding with MSNPs. Binding rate remained constant at subsequent incubation time points. However, maximal binding rates did not change substantially, showing only a marginal increase to approximately $30 \%$ to $35 \%$, which strengthened our assumption that MSNPs preferentially bind to a specific sub-population of sperm, and that targeting with $\mathrm{C} 105 \mathrm{Y}$ perhaps promotes the initial anchoring stage with these cells, rather than facilitating binding to a larger cohort of sperm. Similarly to our previous findings, this binding did not affect sperm function in boar sperm samples. In addition, we observed that the levels of intracellular fluorescence in sperm exposed to MSNPs functionalised with a fluorescent $\mathrm{C} 105 \mathrm{Y}$ 
peptide were higher than those exposed to a free fluorescent $\mathrm{C} 105 \mathrm{Y}$ peptide, which may highlight changes in sperm membrane permeability in the presence of MSNPs.

Collectively, these positive outcomes, demonstrating the biocompatibility of MSNPs with gametes, and the benefits of MSNP functionalization with targeting moieties, have formed the basis for our on-going experiments evaluating the potential of these specialised nanoparticles to transport genetic constructs and proteins into mammalian sperm.. Pilot data for such experiments appears to be encouraging. However, the technique of MSNP-mediated delivery in its current form is associated with many important questions. Firstly, can MSNPs truly internalise into sperm and what are the optimal conditions for this outcome? In our studies, we used the term 'binding' or 'association' to collectively describe the surface attachment of MSNPs to sperm and indicate potential intracellular uptake. There is a growing body of evidence showing that nanomaterials, in fact, do not undergo intracellular uptake into sperm easily: the proportion of nanoparticles reaching the intracellular compartment in sperm after in vitro exposure is remarkably small $[32,40,47]$. In fact, sperm that do internalise nanomaterials appear to exhibit altered integrity of the surface membrane, such as post-acrosome reacted sperm [36]. At the same time, there seems to be a consensus that the internalization of nanocarriers into sperm does not represent a crucial factor for efficient cargo delivery inside the cells, since the performance of nanoparticle-assisted SMGT is not compromised even when the majority of nanoparticles remain attached to the surface membrane [32-34]. Secondly, which properties of sperm predispose such cells to binding with MSNPs and what could be done to increase the binding rates above those that are currently observed? Thirdly, can sperm that have been modified with MSNPs deliver cargo into the oocyte at the time of fertilisation? Finally, what is the fate and effects of exogenous mesoporous silica in mammalian embryos? This is a critical consideration with many potential answers: exclusion, sequestration within a random cell population, or preferential localisation to the embryonic or trophoblastic cells. Given such possibilities, it is vital to investigate how such processes might affect subsequent 
embryo development. Ensuing studies should aim to address these important questions in order to advance our understanding of how nanomaterials may benefit reproductive biology in the future.

\section{CONCLUSIONS}

In summary, our research into the use of MSNPs in boar sperm has taught us some valuable lessons. We now know that MSNPs appear to be biocompatible with boar sperm, and that binding appears to happen preferentially within a specific sperm population with as yet unidentified properties. We also know that the approaches for nanoparticle functionalization that are utilised widely in the field of drug delivery are effective in sperm when a suitable functionalization agent is used. This is highly encouraging given the highly specialised morphology, function and movement patterns in sperm. Nevertheless, even in the case of targeting nanoparticles towards sperm, resultant binding still seems to be largely restricted to a sperm sub-population with an 'inherent affinity' towards MSNPs - a phenomenon requiring further investigation. Our unpublished data also show that MSNPs can deliver exogenous DNA and proteins into boar sperm, and quantification of delivery efficacy, compared to free DNA and protein, forms the next step of our work. We believe that, collectively, our studies show the potential for MSNP-based methodologies in mammalian sperm. However, future studies into the capability of cargo-loaded sperm to deliver molecular agents into oocytes at the time of fertilisation are warranted in order to confirm the end-point efficacy of this novel technique. The encouraging data obtained already from the porcine model in our laboratory, have formed the basis for ethical approval of similar experiments in human sperm, thereby bringing us a step closer towards the potential use of this novel technology in the clinical environment.

\section{FUNDING/ACKNOWLEDGEMENTS}

The authors wish to acknowledge Dr Helen Townley, Dr Christopher Gardiner, Miss Rachel Morrisson and Ms Cindy Huang for their help in synthesising and characterising the nanoparticles. The authors have a patent pending related to the work discussed in this article entitled 'Delivery Method' (PCT Patent Application Number PCT/GB13/053394 filed on the 20th December 2013). Natalia Barkalina is funded by the Clarendon, Scatcherd European and Cyril \& Phillis Long 
Schemes. The project is also funded by the Nuffield Department of Obstetrics and Gynaecology, and an EPSRC Pathways to Impact Award (University of Oxford). The authors have no other relevant affiliations or financial involvement with any organization or entity with a financial interest in, or financial conflict with, the subject matter or materials discussed in the manuscript. This includes employment, consultancies, honoraria, stock ownership or options, expert testimony, grants or patents received or pending, or royalties. No writing assistance was utilized in the production of this manuscript.

\section{FIGURE LEGENDS}

Figure 1. Characterisation of mesoporous silica nanoparticles (MSNPs). (A) Transmission electron microscopy image of unmodified MSNPs. Scale bar $=0.05 \mu \mathrm{m}$; (B) Scanning electron microscopy image of unmodified MSNPs. Scale bar $=0.1 \mu \mathrm{m}$. Synthesised MSNPs were characterized by homogenous size, slightly non-spherical shape with elongation in the direction of the pore channels, and nanometre-sized pores with hexagonal symmetry. Reproduced with permission from [65].

Figure 2. Binding of unloaded MSNPs with sperm. (A) Control; (B) Binding of unloaded MSNPs with sperm. Nanoparticles associated with sperm produced discrete fluorescent signals in various sperm regions (white arrows indicate MSNP-sperm associations). Scale bar $=10 \mu \mathrm{m} .(\mathrm{C}-\mathrm{E})$ Association of unloaded MSNPs with sperm. MSNPs bound to the sperm head and mid-piece. Scale bar $=5 \mu \mathrm{m}$. Reproduced with permission from [65]. 


\section{REFERENCES}

[1] Martin GB. An Australasian perspective on the role of reproductive technologies in world food production. Adv Exp Med Biol. 2014;752:181-97.

[2] Knox RV. Impact of swine reproductive technologies on pig and global food production. Adv Exp Med Biol. 2014;752:131-60.

[3] Galli C, Duchi R, Colleoni S, Lagutina I, Lazzari G. Ovum pick up, intracytoplasmic sperm injection and somatic cell nuclear transfer in cattle, buffalo and horses: from the research laboratory to clinical practice. Theriogenology. 2014;81:138-51.

[4] Holt WV, Brown JL, Comizzoli P. Conclusions: environmental change, wildlife conservation and reproduction. Adv Exp Med Biol. 2014;753:503-14.

[5] Aitken RJ. Age, the environment and our reproductive future: bonking baby boomers and the future of sex. Reproduction. 2014;147:S1-S11.

[6] Gianaroli L, Racowsky C, Geraedts J, Cedars M, Makrigiannakis A, Lobo RA. Best practices of ASRM and ESHRE: a journey through reproductive medicine. Fertil Steril. 2012;98:1380-94.

[7] Freire AG, Resende TP, Pinto-Do-O P. Building and repairing the heart: What can we learn from embryonic development? Biomed Res Int. 2014;10.1155/2014/679168.

[8] Katari R, Peloso A, Zambon JP, Soker S, Stratta RJ, Atala A, et al. Renal bioengineering with scaffolds generated from human kidneys. Nephron Exp Nephrol. 2014;126:119-24.

[9] Felsenstein KM, Candelario KM, Steindler DA, Borchelt DR. Regenerative medicine in Alzheimer's disease. Transl Res. 2014;163:432-8.

[10] Pardal R, Clarke MF, Morrison SJ. Applying the principles of stem-cell biology to cancer. Nat Rev Cancer. 2003;3:895-902.

[11] Kim CF, Dirks PB. Cancer and stem cell biology: How tightly intertwined? Cell Stem Cell. 2008;3:14750.

[12] Harper J, Geraedts J, Borry P, Cornel MC, Dondorp WJ, Gianaroli L, et al. Current issues in medically assisted reproduction and genetics in Europe: research, clinical practice, ethics, legal issues and policy. Hum Reprod. 2014;29:1603-9.

[13] Mueller WA, Hassel M, Grealy M. Development and reproduction in humans and animal model species: Springer; 2015.

[14] Barkalina N, Charalambous C, Jones C, Coward K. Nanotechnology in reproductive medicine: emerging applications of nanomaterials. Nanomedicine. 2014;10:921-38.

[15] Antunes AMD, Alencar MSD, da Silva CH, Nunes J, Mendes FML. Trends in nanotechnology patents applied to the health sector. Recent Pat Nanotech. 2012;6:29-43.

[16] Tsai N, Lee B, Kim A, Yang R, Pan R, Lee D-K, et al. Nanomedicine for global health. JALA. 2014:pii: 2211068214538263.

[17] Serra P, Santamaria P. Nanoparticle-based autoimmune disease therapy. Clin Immunol. 2015;10.1016/j.clim.2015.02.003.

[18] Chrastina A, Massey KA, Schnitzer JE. Overcoming in vivo barriers to targeted nanodelivery. Wiley Interdiscip Rev Nanomed Nanobiotechnol. 2011;3:421-37.

[19] Choudhary S, Kusum Devi V. Potential of nanotechnology as a delivery platform against tuberculosis: Current research review. J Control Release. 2015;202C:65-75.

[20] Singh R, Smitha MS, Singh SP. The role of nanotechnology in combating multi-drug resistant bacteria. J Nanosci Nanotechnol. 2014;14:4745-56.

[21] Nair HB, Sung B, Yadav VR, Kannappan R, Chaturvedi MM, Aggarwal BB. Delivery of antiinflammatory nutraceuticals by nanoparticles for the prevention and treatment of cancer. Biochem Pharmacol. 2010;80:1833-43.

[22] Yin H, Kanasty RL, Eltoukhy AA, Vegas AJ, Dorkin JR, Anderson DG. Non-viral vectors for genebased therapy. Nat Rev Genet. 2014;15:541-55.

[23] Lee N, Choi SH, Hyeon T. Nano-sized CT contrast agents. Adv Mater. 2013;25:2641-60.

[24] Kim TH, Lee S, Chen X. Nanotheranostics for personalized medicine. Expert Rev Mol Diagn. 2013;13:257-69.

[25] Barkalina N, Jones C, Coward K. Mesoporous silica nanoparticles: a potential targeted delivery vector for reproductive biology? Nanomedicine (Lond). 2014;9:557-60.

[26] Gadella BM, Evans JP. Membrane fusions during mammalian fertilization. Adv Exp Med Biol. 2011;713:65-80. 
[27] Jones S, Lukanowska M, Suhorutsenko J, Oxenham S, Barratt C, Publicover S, et al. Intracellular translocation and differential accumulation of cell-penetrating peptides in bovine spermatozoa: evaluation of efficient delivery vectors that do not compromise human sperm motility. Hum Reprod. 2013;28:1874-89.

[28] Huang Z, Wells D. The human oocyte and cumulus cells relationship: new insights from the cumulus cell transcriptome. Mol Hum Reprod. 2010;16:715-25.

[29] Jakop U, Fuchs B, SuSZ R, Wibbelt G, Braun B, Muller K, et al. The solubilisation of boar sperm membranes by different detergents - a microscopic, MALDI-TOF MS, 31P NMR and PAGE study on membrane lysis, extraction efficiency, lipid and protein composition. Lipids Health Dis. 2009;8:49.

[30] Garcia-Vazquez FA, Garcia-Rosello E, Gutierrez-Adan A, Gadea J. Effect of sperm treatment on efficiency of EGFP-expressing porcine embryos produced by ICSI-SMGT. Theriogenology. 2009;72:50618.

[31] Yamauchi Y, Riel JM, Ward MA. Paternal DNA damage resulting from various sperm treatments persists after fertilization and is similar before and after DNA replication. J Androl. 2012;33:229-38.

[32] Kim TS, Lee SH, Gang GT, Lee YS, Kim SU, Koo DB, et al. Exogenous DNA uptake of boar spermatozoa by a magnetic nanoparticle vector system. Reprod Domest Anim. 2010;45:e201-6.

[33] Campos VF, de Leon PMM, Komninou ER, Dellagostin OA, Deschamps JC, Seixas FK, et al. NanoSMGT: Transgene transmission into bovine embryos using halloysite clay nanotubes or nanopolymer to improve transfection efficiency. Theriogenology. 2011;76:1552-60.

[34] Campos VF, Komninou ER, Urtiaga G, de Leon PM, Seixas FK, Dellagostin OA, et al. NanoSMGT: Transfection of exogenous DNA on sex-sorted bovine sperm using nanopolymer. Theriogenology. 2011;75:1476-81.

[35] Makhluf SB, Abu-Mukh R, Rubinstein S, Breitbart H, Gedanken A. Modified PVA-Fe3O4 nanoparticles as protein carriers into sperm cells. Small. 2008;4:1453-8.

[36] Barchanski A, Taylor U, Sajti CL, Gamrad L, Kues WA, Rath D, et al. Bioconjugated gold nanoparticles penetrate into spermatozoa depending on plasma membrane status. J Biomed Nanotechnol. 2015;11.

[37] Odhiambo JF, DeJarnette JM, Geary TW, Kennedy CE, Suarez SS, Sutovsky M, et al. Increased conception rates in beef cattle inseminated with nanopurified bull semen. Biol Reprod. 2014;91:97.

[38] Fynewever TL, Agcaoili ES, Jacobson JD, Patton WC, Chan PJ. In vitro tagging of embryos with nanoparticles. J Assist Reprod Genet. 2007;24:61-5.

[39] Pineda L, Sawosz E, Hotowy A, Elnif J, Sawosz F, Ali A, et al. Effect of nanoparticles of silver and gold on metabolic rate and development of broiler and layer embryos. Comp Biochem Physiol A Mol Integr Physiol. 2012;161:315-9.

[40] Feugang JM, Youngblood RC, Greene JM, Fahad AS, Monroe WA, Willard ST, et al. Application of quantum dot nanoparticles for potential non-invasive bio-imaging of mammalian spermatozoa. J Nanobiotechnology. 2012;10:45.

[41] Lavitrano M, Bacci ML, Forni M, Lazzereschi D, Di Stefano C, Fioretti D, et al. Efficient production by sperm-mediated gene transfer of human decay accelerating factor (hDAF) transgenic pigs for xenotransplantation. Proc Natl Acad Sci USA. 2002;99:14230-5.

[42] Eghbalsaied S, Ghaedi K, Laible G, Hosseini SM, Forouzanfar M, Hajian M, et al. Exposure to DNA is insufficient for in vitro transgenesis of live bovine sperm and embryos. Reproduction. 2013;145:97-108.

[43] Wu Y, Liu CJ, Wan PC, Hao ZD, Zeng SM. Factors affecting the efficiency of producing porcine embryos expressing enhanced green fluorescence protein by ICSI-mediated gene transfer method. Anim Reprod Sci. 2009;113:156-66.

[44] Garcia-Vazquez FA, Ruiz S, Grullon LA, de Ondiz A, Gutierrez-Adan A, Gadea J. Factors affecting porcine sperm mediated gene transfer. Res Vet Sci. 2011;91:446-53.

[45] Coward K, Kubota H, Parrington J. In vivo gene transfer into testis and sperm: developments and future application. Arch Androl. 2007;53:187-97.

[46] Rath D, Barcikowski S, de Graaf S, Garrels W, Grossfeld R, Klein S, et al. Sex selection of sperm in farm animals: status report and developmental prospects. Reproduction. 2013;145:R15-30.

[47] Taylor U, Barchanski A, Petersen S, Kues WA, Baulain U, Gamrad L, et al. Gold nanoparticles interfere with sperm functionality by membrane adsorption without penetration. Nanotoxicology. 2014;8 Suppl 1:11827.

[48] Barchanski A, Taylor U, Klein S, Petersen S, Rath D, Barcikowski S. Golden perspective: application of laser-generated gold nanoparticle conjugates in reproductive biology. Reprod Domest Anim. 2011;46 Suppl $3: 42-52$. 
[49] Taylor U, Petersen S, Barchanski A, Mittag A, Barcikowski S, Rath D. Influence of gold nanoparticles on vitality parameters of bovine spermatozoa. Reprod Domest Anim. 2010;45:60.

[50] Amann RP. Use of animal models for detecting specific alterations in reproduction. Fundam Appl Toxicol. 1982;2:13-26.

[51] Working PK. Male reproductive toxicology: comparison of the human to animal models. Environ Health Perspect. 1988;77:37-44.

[52] Quinn P, Horstman FC. Is the mouse a good model for the human with respect to the development of the preimplantation embryo in vitro? Hum Reprod. 1998;13 Suppl 4:173-83.

[53] Menezo YJ, Herubel F. Mouse and bovine models for human IVF. Reprod Biomed Online. 2002;4:1705.

[54] Amdani SN, Jones C, Coward K. Phospholipase C zeta (PLCzeta): oocyte activation and clinical links to male factor infertility. Adv Biol Regul. 2013;53:292-308.

[55] Tardif S, Madamidola OA, Brown SG, Frame L, Lefievre L, Wyatt PG, et al. Clinically relevant enhancement of human sperm motility using compounds with reported phosphodiesterase inhibitor activity. Hum Reprod. 2014;29:2123-35.

[56] Vallet-Regi M, Ramila A, del Real RP, Perez-Pariente J. A new property of MCM-41: Drug delivery system. Chem Mater. 2001;13:308-11.

[57] Vallet-Regi M, Balas F, Arcos D. Mesoporous materials for drug delivery. Angew Chem Int Edit. 2007;46:7548-58.

[58] Gan Q, Dai D, Yuan Y, Qian J, Sha S, Shi J, et al. Effect of size on the cellular endocytosis and controlled release of mesoporous silica nanoparticles for intracellular delivery. Biomed Microdevices. 2012;14:259-70.

[59] Brinker CJ, Scherer GW. Sol-gel science: the physics and chemistry of sol-gel processing. Boston; London: Academic Press; 1990.

[60] Coti KK, Belowich ME, Liong M, Ambrogio MW, Lau YA, Khatib HA, et al. Mechanised nanoparticles for drug delivery. Nanoscale. 2009;1:16-39.

[61] Wu SH, Hung Y, Mou CY. Mesoporous silica nanoparticles as nanocarriers. Chem Commun. 2011;47:9972-85.

[62] Rosenholm JM, Mamaeva V, Sahlgren C, Linden M. Nanoparticles in targeted cancer therapy: mesoporous silica nanoparticles entering preclinical development stage. Nanomedicine (Lond). 2012;7:11120.

[63] Kunzmann A, Andersson B, Thurnherr T, Krug H, Scheynius A, Fadeel B. Toxicology of engineered nanomaterials: focus on biocompatibility, biodistribution and biodegradation. Biochim Biophys Acta. 2011;1810:361-73.

[64] Xia T, Kovochich M, Liong M, Meng H, Kabehie S, George S, et al. Polyethyleneimine coating enhances the cellular uptake of mesoporous silica nanoparticles and allows safe delivery of siRNA and DNA constructs. ACS Nano. 2009;3:3273-86.

[65] Barkalina N, Jones C, Kashir J, Coote S, Huang X, Morrison R, et al. Effects of mesoporous silica nanoparticles upon the function of mammalian sperm in vitro. Nanomedicine. 2014;10:859-70.

[66] Barkalina N, Charalambous C, Jones C, Coward K. Mesoporous silica nanoparticles in mammalian sperm: effects of in vitro exposure upon fertilization potential [oral presentation]. BioNanoMed 2014 - 5th International Congress on Nanotechnology, Medicine and Biology. 2014;10.13140/2.1.3738.7847.

[67] Barkalina N, Jones C, Townley H, Coward K. Functionalisation of mesoporous silica nanoparticles with a cell-penetrating peptide to target mammalian sperm in vitro. Nanomedicine (Lond). 2015;[In press]. 